\title{
Projeto de compensadores pelo método da resposta de frequência: Uma interface interativa
}

\author{
Magno Enrique Mendoza Meza ${ }^{1}$ \\ ${ }^{1}$ Universidade Federal do ABC \\ Centro de Engenharia, Modelagem e Ciências Sociais Aplicadas (CECS) \\ Curso de Mestrado em Engenharia Mecânica (PosMEC) \\ Av. dos Estados 5001, Bloco A, Sala 744-1, SP09210-580 \\ Bangu, Santo André, São Paulo, Brasil \\ magno.meza@ufabc.edu.br
}

\begin{abstract}
In this work shows an user interface interactive for the design of different types of compensators (lead, lag and jag-lead phase) by the frequency response method. This method belongs to the program of study in different engineering courses. The interface was implemented in MATLAB/GUIDE. The purpose of this interface is to assist engineering students in the study, analysis and design of these compensators and develop their engineering abilities through lab works. The interface shows useful due to in each step of the procedure design the performance parameters of the system are shown, as well as the effort control amplitude.
\end{abstract}

Resumo. Neste trabalho apresenta-se uma interface de usuário interativa para o projeto de diferentes tipos de compensadores (por avanço, por atraso e por atraso-avanço de fase) utilizando o método da resposta de frequência. Esse método faz parte do programa de estudo nos diferentes cursos de engenharia. A interface foi implementada no MATLAB/GUIDE. O propósito dessa interface é auxiliar os alunos de engenharia no estudo, análise e projeto desses tipos de compensadores e desenvolver as suas habilidades através dos trabalhos laboratoriais. A interface mostra-se útil porque a cada passo dos projetos os parâmetros de desempenho do sistemas em estudo são mostrados, bem como a amplitude do esforço de controle.

\section{Introdução}

O ensino laboratorial na área de controle auxiliado por computadores é um fato na atualidade; os computadores ajudam no processo de aprendizagem dos alunos com as simulações, metodologia de projeto dos compensadores e análise dos sistemas de controle. Novos métodos e práticas pedagógicas constituem um grande desafio para os educadores nas áreas de ensino da engenharia de controle. O maior desafio no ensino da teoria de sistemas de controle é a necessidade de capacitar os alunos com uma base teórica forte e habilidades em engenharia. A base teórica pode ser lecionada em uma sala de aula normal, mas as habilidades em engenharia podem ser obtidas com trabalhos laboratoriais [Johansson et al. 1998, Garcia and Heck 1999, Haffner et al. 2006]. Nos últimos trinta anos o cenário do ensino da teoria de sistemas de controle está baseada no desenvolvimento de ferramentas interativas utilizando o 
MATLAB [Garcia and Heck 1999, Savaş and Erdal 2010, Johansson et al. 1998, Dormido et al. 2005, Guzman et al. 2013, Sánchez et al. 2005, Haffner et al. 2006], Java [Méndez et al. 2006], LabView [Stefanovic et al. 2009], C/C++ [Chen et al. 2010], MATHEMATICA [Kujan et al. 2005], Maple e MATLAB [Da̧browski et al. 2011].

O MATLAB é um ambiente de programação para o desenvolvimento de algoritmos, análise de dados, visualização e cálculo numérico. Pode-se usar o MATLAB em uma ampla gama de aplicações, tais como processamento de sinais e de imagem, comunicação, modelagem e análise financeira e biologia computacional, bem como no projeto de sistemas de controle. Sendo uma linguagem computacional técnica, muitos engenheiros e cientistas na indústria e na academia utilizam o MATLAB, o qual permite criar interfaces gráficas do usuário (GUI - Graphical User Interfaces), que estão compostas por uma janela do tipo Figura contendo menus, textos, botões, gráficos, etc., os quais o usuário pode manipular de forma interativa através do mouse e do teclado [Marchand and Holland 2003].

O Prof. Erik Cheever da Swarthmore College [Cheever and Li 2005] desenvolveu vários programas entre os quais está o BodePlotGui.m, que é uma interface gráfica escrita na linguagem de programação MATLAB/GUIDE. Este programa tem como entrada uma função de transferência e a decompõe em fatores elementais, e assim, desenha as linhas assintóticas de cada elemento. Esse programa é versátil para o ensino e aprendizagem no desenho do diagrama de Bode por partes das aproximações lineares. Termos individuais podem ser adicionados ou excluídos do diagrama de Bode. Este programa está disponíveis na site da MathWorks.

Disciplinas de sistemas de controle fazem parte do currículo de diversos cursos de engenharia e tem como objetivo fixar conceitos de análise de sistemas de controle e projeto de controladores. Os controladores são utilizados em diversas áreas como automação de processos, na indústria, etc. Um dos métodos de projeto de controladores é o da resposta em frequência [Ogata 2008, Dorf and Bishop 2011, Nise 2000], que faz parte das disciplinas de sistemas de controle.

Um sistema de controle adequado deve ser estável, com uma resposta aceitável às excitações de entrada e erro de estado estacionário pequeno. A compensação é uma forma de obter um desempenho adequado de um sistema de controle alterando a forma da sua resposta em frequência. Um compensador é um componente inserido em um sistema de controle para compensar as deficiências de desempenho [Dorf and Bishop 2011]. A função do compensador por avanço de fase é mudar a curva de resposta em frequência para obter um ângulo de avanço de fase suficiente para compensar o excessivo atraso de fase do sistema. A função do compensador por atraso de fase é produzir atenuação nas altas frequências para oferecer ao sistema uma margem de fase suficiente. A compensação por atraso-avanço de fase combina as características do compensador por atraso e do compensador por avanço de fase.

Neste trabalho, apresenta-se uma interface de usuário interativa de simulação e projeto de compensadores para sistemas de controle lineares de uma entrada e uma saída pelo método da resposta em frequência. Os compensadores mostrados nesta interface são: por avanço de fase, por atraso de fase e por atraso-avanço de fase. 
Os projetos desses compensadores estão baseadas nos procedimentos mostrados em Ogata [Ogata 2008]. Este trabalho está organizado da seguinte maneira: na seção 2 é apresentado de maneira resumida o conceito da resposta em frequência; na Seção 3 é mostrado o procedimento de projeto de um compensador por avanço de fase; na Seção 4 é explicada a interface de usuário interativa do projeto do compensador por avanço de fase; na Seção 5 são mostradas as Conclusões e Discussões do trabalho apresentado. Por falta de espaço somente é mostrada a interface interativa do compensador por avanço de fase.

\section{Resposta em frequência}

Os métodos de resposta em frequência foram desenvolvidos nos anos de 1930 e 1940 com H. Nyquist [Nyquist 1932] e H. W. Bode [Bode 1940]. A resposta em frequência produz um enfoque vantajoso a partir do qual pode-se tratar os sistemas de controle com realimentação. Esse método tem vantagem nas seguintes situações:

1. Na modelagem das funções de transferência a partir de dados físicos;

2. No projeto de compensadores de avanço de fase para satisfazer requerimentos de erros de estado estacionário e resposta transitória;

3. Na determinação da estabilidade de sistemas não lineares.

No estado estacionário, entradas senoidais aplicadas a sistemas lineares geram respostas senoidais da mesma frequência que a entrada (excitação), mas de amplitude e ângulo de fase diferentes, que são funções da frequência, veja Figura 1(a). A representação gráfica da resposta em frequência é muito útil e uma dessas representações são chamadas de diagramas de Bode. O diagrama de Bode consiste em um gráfico duplo, o primeiro é o gráfico do ganho em dB e o segundo do ângulo de fase e ambos em função da frequência $\omega$ em escala logarítmica. Esses gráficos são importantes pois são úteis na análise de desempenho, no projeto de compensadores e na identificação de sistemas.

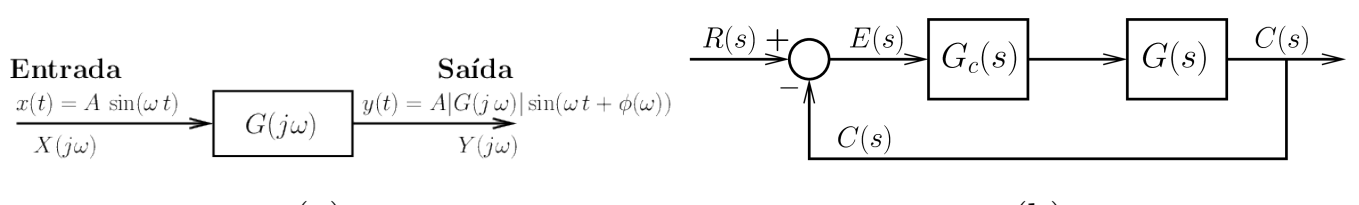

(a)

(b)

Figura 1. (a) Resposta em frequência. (b) Diagrama de blocos do sistema de controle com compensador em cascata.

Considere-se um sistema com realimentação negativa com uma função de transferência em malha aberta representada por $G(s)$, e uma função de transferência do compensador em cascata representado por $G_{c}(s)$, veja Figura 1(b).

\section{Compensador por avanço de fase: Procedimento de projeto}

A resposta em frequência da estrutura de compensação em cascata, $G_{c}(s)$, é adicionada à resposta em frequência do sistema não compensado, $G(s)$, isto é, a função de transferência de malha aberta é dada por $G_{c}(s) G(s)$, veja Figura 1(b). O procedimento de projeto de um compensador por avanço de fase pelo método da resposta em frequência é mostrado a seguir: 
1. Obtenha o diagrama de Bode do sistema sem compensação com o ganho $K_{1}$, i.e., $G_{1}(s)=K_{1} G(s)$, ajustado para que a constante de erro estático desejada seja satisfeita;

2. Determine a margem de fase do sistema sem compensação com o ganho ajustado, e se ela for insuficiente, prossiga para o passo a seguir;

3. Se a margem de fase não for suficiente pode-se adicionar uma avanço de fase. Determine o ângulo de avanço de fase necessário que o compensador deve contribuir ao sistema;

4. Calcule o valor do fator de atenuação $\alpha$. Defina a frequência de forma que o módulo do sistema sem compensação com o ganho ajustado satisfaça $G_{1}(j \omega)=-20 \log (1 / \sqrt{\alpha})$. Selecione essa frequência como a nova frequência de cruzamento de ganho, que corresponde a $\omega_{m}=\frac{1}{\sqrt{\alpha} T}$ e a defasagem máxima $\phi_{m}$ ocorrerá nessa frequência;

5. Determine as frequências de canto do compensador correspondente ao polo e ao zero;

6. Traçar o diagrama de Bode da resposta em frequência compensada. Verifique se a margem de ganho é satisfatório. Caso contrário, repita o procedimento modificando a localização do polo e do zero do compensador.

\section{Interface de usuário interativa: Projeto do compensador por avanço de fase}

A Figura 2(a) mostra a interface gráfica do usuário. Nesta janela é possível selecionar o tipo de compensador que será projetado e simulado. Na interface estão disponíveis os seguintes compensadores: Avanço, Atraso e Atraso-Avanço de fase. Ao clicar em alguma das opções o usuário terá acesso a uma nova interface com caixa de entrada e saída de dados. A Figura 2(b) mostra a interface de usuário para o projeto e simulação do compensador por avanço de fase. Considere, como exemplo, que o

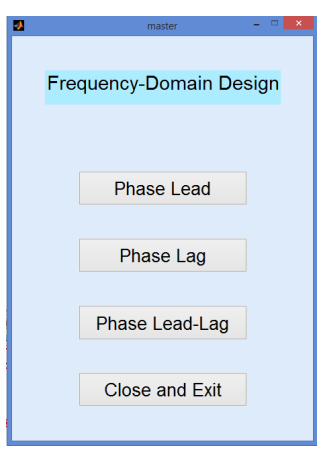

(a)

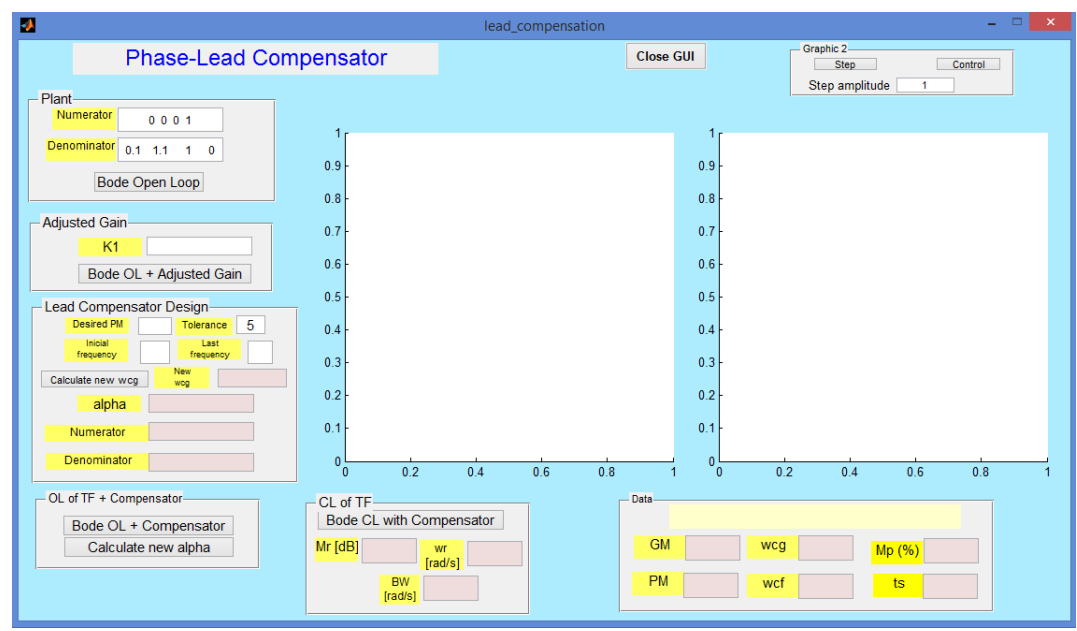

(b)

Figura 2. (a) Interface gráfica principal do usuário para o projeto dos compensadores no domínio da frequência, arquivo master.m. (b) Interface gráfica interativa para o projeto do compensador por avanço de fase. 
sistema de malha aberta mostrado na Figura $1(\mathrm{~b})$ tem $G(s)=\frac{1}{s(0,1 s+1)(s+1)}$. Deseja-se projetar um compensador por avanço de fase $G_{c}(s)$, de modo que a margem de fase desejada seja de $45^{\circ}$, a margem de ganho não seja inferior a $8 \mathrm{~dB}$ e a constante de erro estático de velocidade $K_{v}$ seja de $4 \mathrm{~s}^{-1}$.

Para iniciar o programa, na janela de comandos do MATLAB, deve-se definir a variável MySys como uma função de transferência, definindo também seu numerador e denominador, por exemplo,

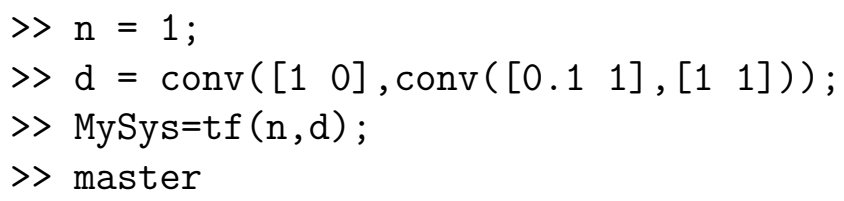

Executado esses comandos, o MATLAB abre a interface gráfica mostrada na Figura 2(a) e ao clicar na opção "Phase Lead" é aberto a interface gráfica mostrada na Figura 2(b).

A Figura 3(a) mostra a painel utilizado para introduzir os coeficientes do numerador e denominador da função de transferência da planta em malha aberta. Ao clicar o botão "Bode Open Loop", é desenhado (veja Figura 5) no primeiro eixo, o diagrama de Bode e no segundo, a resposta do sistemas em malha fechada a uma entrada degrau unitária e no painel "Data" (veja Figura 4) são mostrados os valores dos parâmetros de desempenho do sistema. A Figura 3(b) mostra o painel "Adjusted Gain" utilizado para introduzir o valor do ganho $K_{1}$ que satisfaz algumas das constantes de erro estáticos; o aluno deve determinar o valor do ganho utilizando o conceito correspondente à constate de erro estático. Ao clicar o botão "Bode OL + Adjusted Gain" é plotado o diagrama de Bode do sistema sem compensar mas com ganho ajustado, bem como a resposta à entrada degrau unitário nestas condições (veja Figura 5). A Figura 3(c) mostra o painel "Lead Compensator Design", no qual o usuário pode especificar a margem de fase desejada (PM) e a tolerância necessária para o projeto, ambos valores em graus, bem como as respectivas expoentes da frequência mínima e máxima do diagrama de Bode. Ao clicar o botão "Calculate new $\omega_{c g}$ " são mostrado os correspondentes valores da nova frequência $\omega_{c g}$, o valor do fator de atenuação $\alpha$ (alpha), os coeficientes do numerador e denominador do compensador por avanço de fase.

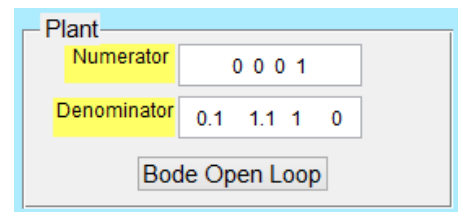

(a)

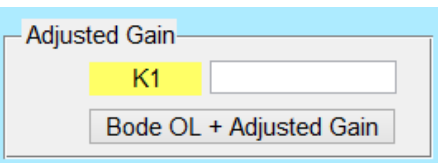

(b)

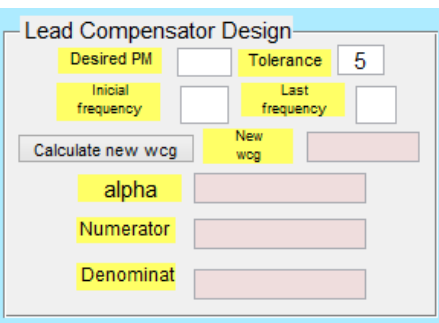

(c)

Figura 3. (a) Painel "Plant". (b) Painel “Adjusted Gain". (c) Painel "Lead Compensator Design". 


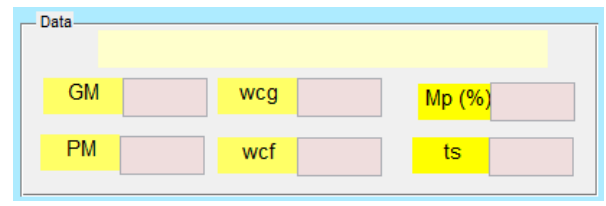

Figura 4. Painel “Data”.

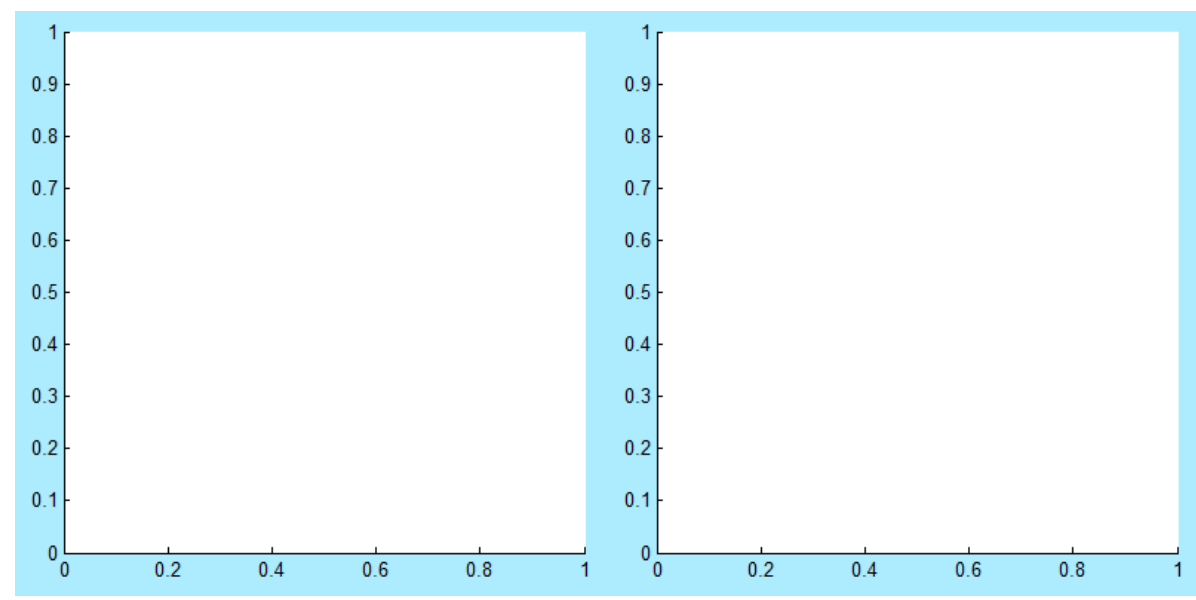

Figura 5. Eixos para desenhar o diagrama de Bode e a resposta à entrada degrau unitária.

A Figura 6(a) mostra o painel "OL of TF + Compensator", ao clicar o botão "Bode OL + Compensator" é plotado o diagrama de Bode do sistema compensado e a resposta a uma entrada degrau unitária ou a amplitude do esforço de controle, e os valores dos parâmetros de desempenho são mostrados no painel "Data" (veja Figura 4). Ao clicar o botão "Calculate new alpha" serão determinados novos valores de $\omega_{c g}$, numerador e denominador do compensador para uma nova escolha de $\alpha$. A Figura 6(b) mostra o painel "CL of TF", ao clicar no botão "Bode CL with Compensator" serão mostrados no respectivo painel o valor do pico de ressonância $M_{r}$ em dB, a frequência de ressonância $\omega_{r} \mathrm{em} \mathrm{rad} / \mathrm{s}$ e a largura de banda $B W \mathrm{em} \mathrm{rad} / \mathrm{s}$.

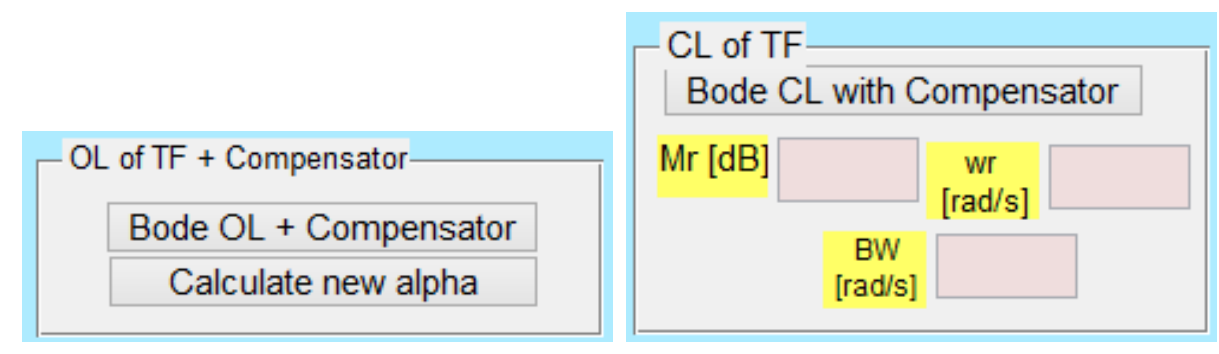

(a)

(b)

Figura 6. (a) Painel "OL of TF + Compensator". (b) Painel "CL of TF".

A Figura 7 mostra o botão "Close GUI" que ao ser clicado fecha a respectiva interface e retorna à interface principal, e o painel "Graphic 2" que ao clicar o botão "Step" será plotada a respectiva resposta do sistema a uma entrada degrau unitária de amplitude determinada pelo valor na caixa de entrada "Step amplitude" e ao 


\begin{tabular}{|c|c|c|}
\hline \multirow{3}{*}{ Close GUI } & \multicolumn{2}{|l|}{ - Graphic 2} \\
\hline & Step & \begin{tabular}{|l|} 
Control \\
\end{tabular} \\
\hline & Step amplitude & 1 \\
\hline
\end{tabular}

Figura 7. Painel "Graphic 2" e o botão "Close GUI”.

clicar no botão "Control" será plotado a amplitude do esforço de controle necessária para a respectiva entrada ao degrau de amplitude "Step amplitude".

A Figura 8 mostra a interface após concluído o procedimento de projeto do compensador por avanço de fase para o exemplo dado.

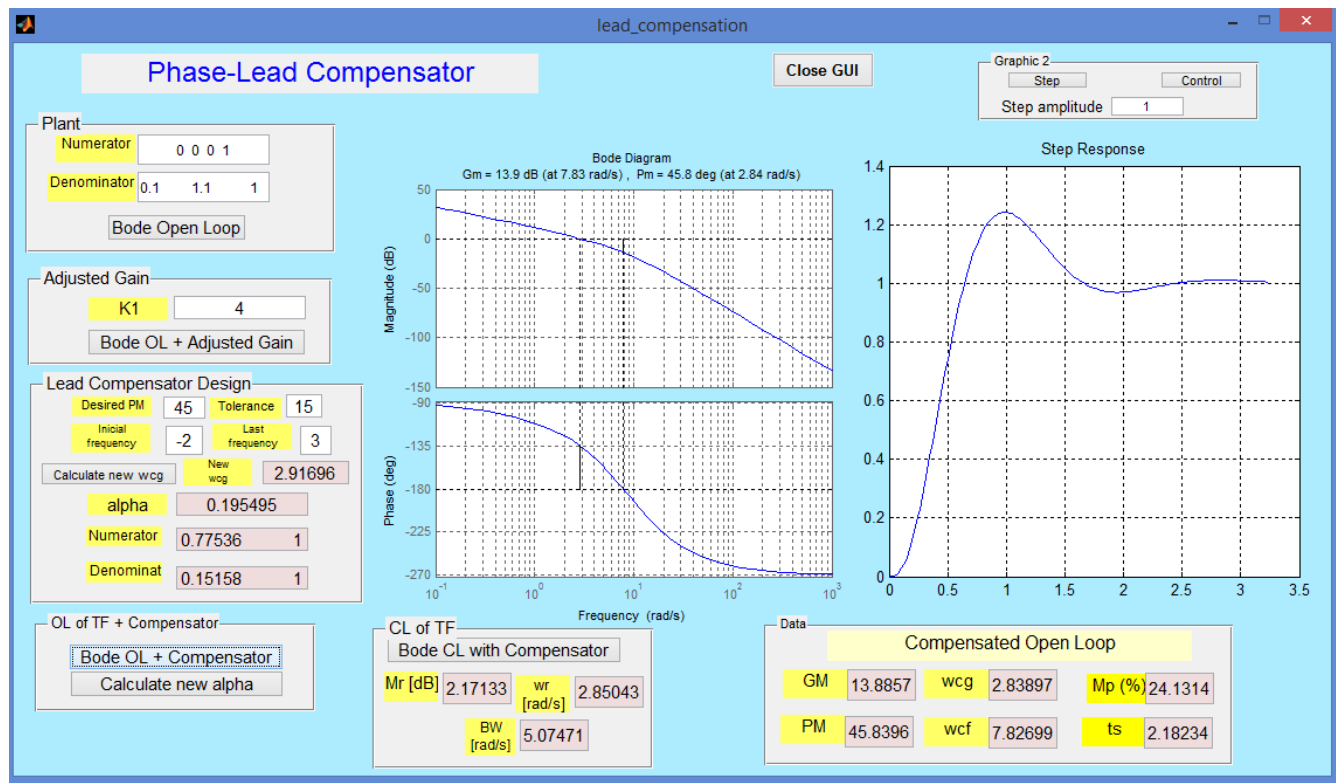

Figura 8. Interface interativa para o projeto do compensador por avanço de fase no domínio da frequência. Mostra-se o diagrama de Bode do sistema de controle compensado em malha aberta e a resposta do sistema a uma entrada degrau unitária.

A Figura 9 mostra a interface após concluído o procedimento de projeto do compensador por avanço de fase para o exemplo dado, no qual é mostrado o esforço de controle necessário para o sistema compensado.

\section{Conclusões e Discussões}

Neste trabalho mostra-se uma interface gráfica do usuário baseada no MATLAB/GUIDE para o projeto e simulação de diferentes tipos de compensadores pelo método da resposta em frequência. O propósito desta interface didática é auxiliar os alunos de engenharia no estudo, análise e projeto desses tipos de compensadores.

A programação da interface do usuário é um conjunto de "scripts" feitos na linguagem do MATLAB, mas uma das características da interface é a interatividade, pois o usuário pode introduzir novos valores de parâmetros de projeto e executar novamente a interface evitando-se, assim, executar os conjuntos de "scripts" de maneira independente, bem como é possível observar os diferentes valores dos parâmetros de desempenho para cada modificação feita no projeto dos compensadores. Com esta 


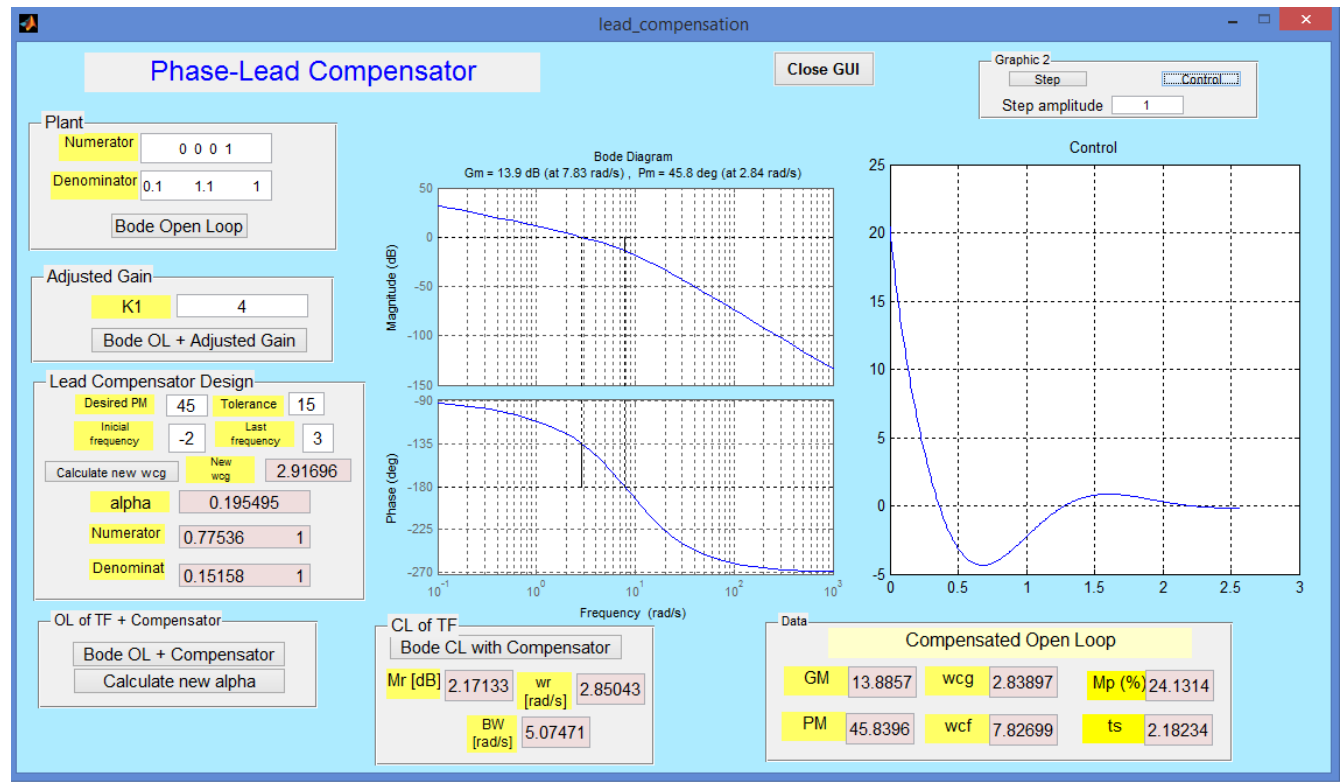

Figura 9. Interface interativa para o projeto do compensador por avanço de fase no domínio da frequência. Mostra-se o diagrama de Bode do sistema de controle compensado em malha aberta e o esforço de controle aplicado na planta devido a uma entrada degrau unitária.

interface o aluno se concentrará nos conceitos teóricos ao invés de gastar tempo desenvolvendo tarefas computacionais e assim reforçará o entendimento dos conceitos teóricos com as simulações e a interatividade da interface.

Os procedimentos de projeto dos compensadores podem ser achados em qualquer livro de sistemas de controle [Dorf and Bishop 2011, Ogata 2008, Nise 2000].

A interface didática está na sua primeira versão e espera-se a contribuição dos alunos e professores da área de sistemas de controle para que a mesma possa ser aperfeiçoada. No último quadrimestre de 2014 a interface didática será disponibilizada entre a comunidade discente da disciplina de Sistemas de Controle II da Universidade Federal do ABC com o intuito de ser testada e avaliada pelos discentes. Para um futuro trabalho, pretende-se implementar esta interface gráfica utilizando software livre e, assim, poderá ser utilizada sem a necessidade do MATLAB.

\section{Referências}

Bode, H. W. (1940). Relations between attenuation and phase in feedback amplifier design. Bell System Technical Journal, 19(3):421-454.

Cheever, E. and Li, Y. (2005). A tool for construction of bode diagrams from piecewise linear asymptotic approximations*. Int. J. Engng Ed., 21(2):335-340.

Chen, B., Chou, Y.-C., and Cheng, H. H. (2010). Open source ch control system toolkit and web-based control system design for teaching automatic control of linear time-invariant systems. Computer Applications in Engineering Education, 47:95-112.

Dạbrowski, A., Mitkowski, S. A., and Porȩbska, A. (2011). The use of mathematical programs and numerical methods in teaching selected topics in circuit 
theory based on Maple and MATLAB. Global Journal of Engineering Education, 13(3):132-139.

Dorf, R. C. and Bishop, R. H. (2011). Sistemas de Controle Modernos. LTC, Rio de Janeiro, 11a. edition.

Dormido, S., Dormido-Canto, S., Dormido, R., Sánchez, J., and Duro, N. (2005). The role of interactivity in control learning. Int. J. Engng. Ed., 21(6):1122-1133.

Garcia, R. C. and Heck, B. S. (1999). Enhacing classical controls education via interactive GUI design. IEEE Control Systems, 19(3):77-82.

Guzman, J. L., Dormido, S., and Berenguel, M. (2013). Interactivity in education: An experience in the automatic control field. Computer Applications in Engineering Education, 21(2).

Haffner, J. F., Pereira, L. F. A., and Coutinho, D. F. (2006). Computer-assisted evaluation of undergraduate courses in frequency-domain techniques for system control. IEEE Trans. on Education, 49(2):224-235.

Johansson, M., Gäfvert, M., and Åström, K. J. (1998). Interactive tools for education in automatic control. IEEE Control Systems Magazine, 18(3):33-40.

Kujan, P., HromčíK, M., and Šebek, M. (2005). Web-based MATHEMATICA platform for system and control education. In Proceedings of the 13th Mediterranean Conference on Control and Automation, pages 376-381. IEEE.

Marchand, P. and Holland, O. T. (2003). Graphics and GUIs with MATLAB. Chapman \& Hall/CRC, Florida, third edition.

Méndez, J. A., Lorenzo, C., Acosta, L., Torres, S., and González, E. (2006). A webbased tool for control engineering teaching. Computer Applications in Engineering Education, 14(3):178-187.

Nise, N. S. (2000). Engenharia de Sistemas de Controle. LTC, Brasil.

Nyquist, H. (1932). Regeneration theory. Bell System Technical Journal, 11(1):126147.

Ogata, K. (2008). Engenharia de Controle Moderno. Pearson \& Prentice Hall, Brasil.

Sánchez, J., Dormido, S., and Esquembre, F. (2005). The learning of control concepts using interactive tools. Computer Applications in Engineering Education, 13(1):84-98.

Savaş, K. and Erdal, H. (2010). Automatic control simulation environment system (ACSES) designed as a virtual tool for control education. Procedia Social and Behavioral Sciences, 2(2):5233-5237.

Stefanovic, M., Cvijetkovic, V., Matijevic, M., and Simic, V. (2009). A LabVIEWbased remote laboratory experiments for control engineering education. Computer Applications in Engineering Education, 19:538-549. 